\title{
Author Correction: The complexity of the Fragaria x ananassa(octoploid) transcriptome by single-molecule long-read sequencing
}

Huazhao Yuan', Hongmei Yu' ${ }^{1}$ Tao Huang ${ }^{2}$, Xinjie Shen ${ }^{3}$, Jin Xia ${ }^{1}$, Fuhua Pang ${ }^{1}$, Jing Wang ${ }^{1}$ and Mizhen Zhao ${ }^{1}$

Correction to: Horticulture Research (2019) 6, 46 https://doi.org/10.1038/s41438-019-0126-6 published online 01 April 2019

Since the publication of this article, the authors have noticed that the part of acknowledgement is missing from article. Here is the acknowledgement:
This work was supported by the National Key Research and Development Program of China (2018YFD1000300), National Crop Germplasm Resources Protection of Ministry of Agriculture of China (NB2018-2130135-07).

The authors would like to apologize for this error.

Published online: 19 April 2019

Correspondence: Mizhen Zhao (njzhaomz@163.com) 